\title{
Monolithic Interconnected Modules (MIMs) for Thermophotovoltaic Energy Conversion
}

\author{
David Wilt ${ }^{1}$, Rebecca Wehrer ${ }^{2}$, Marc Palmisiano $^{2}$, Mark Wanlass ${ }^{3}$ and Christopher \\ Murray $^{4}$ \\ ${ }^{1}$ NASA Glenn Research Center, Cleveland, OH USA \\ ${ }^{2}$ Bechtel Bettis Inc, West Mifflin, PA USA \\ ${ }^{3}$ NREL, Golden, CO USA \\ ${ }^{4}$ Emcore Photovoltaics, Albuquerque, NM USA
}

\begin{abstract}
Monolithic Interconnected Modules (MIM) are under development for thermophotovoltaic (TPV) energy conversion applications. MIM devices are typified by series-interconnected photovoltaic cells on a common, semi-insulating substrate and generally include rear-surface infrared (IR) reflectors. The MIM architecture is being implemented in InGaAsSb materials without semi-insulating substrates through the development of alternative isolation methodologies. Motivations for developing the MIM structure include: reduced resistive losses, higher output power density than for systems utilizing front surface spectral control, improved thermal coupling and ultimately higher system efficiency. Numerous design and material changes have been investigated since the introduction of the MIM concept in 1994. These developments as well as the current design strategies are addressed.
\end{abstract}

\section{Introduction}

Thermophotovoltaic (TPV) energy conversion is a direct thermal-to-electric energy conversion process whereby an emitter or radiator is heated to incandescence and a photovoltaic device is placed in view of the emitter. Incident photons of sufficient energy are converted into electrical energy by the photovoltaic device. High efficiency TPV systems require highly efficient spectral control in the cavity formed by the emitter and the photovoltaic array. In an ideal TPV system, the low-bandgap photovoltaic device can absorb only photons with energies greater than the device bandgap. All other photons would either not be emitted at all, or they would be reabsorbed by the emitter for full thermal recovery. For efficient operation, up to $75 \%$ of the radiant energy from the emitter needs to be recycled for a TPV system with a moderate temperature broadband emitter (1200K to $1500 \mathrm{~K})$ and a low-bandgap photovoltaic device $(0.5 \mathrm{eV}<\mathrm{Eg}<0.75$ $\mathrm{eV})$. This emphasizes the tremendous importance of spectral control in obtaining high TPV system efficiencies. Spectral control can be implemented on the emitter, on the front or rear surfaces of the photovoltaic device, or on a combination of these locations.

Photovoltaic devices developed for most TPV applications have had bandgaps ranging from $0.5 \mathrm{eV}$ to $0.75 \mathrm{eV}$. Generally speaking, these low-bandgap devices produced high current densities and low voltages. These operating characteristics, combined with the need for efficient spectral control, were the impetus for the development of the first MIM concept that monolithically integrated spectral control into the power conversion device 
[1]. This MIM concept utilized the cell interconnect architecture previously demonstrated for laser power conversion [2]. The MIM device originally consisted of series-connected $\mathrm{p} / \mathrm{n}$ indium gallium arsenide (InGaAs) devices on a common semiinsulating indium phosphide (InP) substrate (figure 1). The semi-insulating InP substrate provided two key advantages: Electrical isolation for the component cells and near perfect optical transparency for sub-bandgap photons. This last characteristic allowed the inclusion of an infrared (IR) mirror deposited on the polished back surface of the InP substrate to reflect unabsorbed photons back toward the front surface of the cell. Thus the MIM design integrates the energy conversion and spectral control functions into a single device.

The MIM design offers several advantages. Firstly, small series-connected cells provide high voltages and low currents, reducing Joule losses. In addition, a MIM array may be comprised of series/parallel strings rather than a single series-connected string of large cells. This should improve the reliability of the TPV module since the failure of a single cell would not debilitate the entire array. Secondly, the design should enable high output power densities since losses associated with front-surface spectral controls may be eliminated. Thirdly, the rear surface of the device is not electrically active therefore the cell may be directly bonded to the substrate/heat sink without concern for electrical isolation. This greatly simplifies the array design and improves the thermal control of the cells. Lastly, photons that are weakly absorbed have the possibility of multiple passes through the cell structure. This feature is particularly important for devices with low minority carrier diffusion length due to either lattice-mismatch or radiation degradation.

\section{InGaAs MIM}

In the current and original MIM designs, a continuous layer of metallization connects cells in series by connecting the lateral conduction layer (LCL) of one cell with the emitter of the adjacent cell. This has been termed the buss bar interconnect. A layer of dielectric is deposited prior to the interconnect deposition to protect the exposed junction edge from being shorted by the interconnect. This geometry is particularly effective for the III-V materials where the cells are defined and electrically isolated by anisotropic wet chemical etching. With the correct crystallographic orientation, the interconnects are deposited in the $\mathrm{v}$-groove trench rather than the dovetail trench.

The design of any MIM device requires a balance between designing for optimum electrical and optical efficiency. As shown in figure 1, the original MIM design utilized a $\mathrm{p} / \mathrm{n}$ configuration. This polarity offers some electrical advantages compared to the $\mathrm{n} / \mathrm{p}$ configuration. Given the tremendous difference in carrier mobility $(25 \mathrm{x})$ between $\mathrm{n}$ and $\mathrm{p}$ type InGaAs, the resistive losses associated with lateral current conduction are dramatically reduced in the $2 \times 10^{19} / \mathrm{cm}^{3} \mathrm{n}++$ conductor layer ( $\mathrm{LCL}$ - lateral conduction layer). In addition, heavily $\mathrm{n}$-doped $\mathrm{InGaAs}$ demonstrates a Burstein-Moss shift (figure 2) due to filling of states near the bottom of the conduction band $[3,4]$. Thus for the same composition (no change in lattice parameter), the $\mathrm{n}++$ InGaAs LCL behaves like a transparent conducting layer. This feature allows the base of the device to be grown 
intentionally thin, thereby allowing weakly absorbed photons a second pass opportunity and forcing the photo generation of carriers to occur closer to the $\mathrm{p} / \mathrm{n}$ junction.

The data in figure 2 also demonstrate a problem with the use of highly doped p-type material, namely a significant increase in free carrier absorption. Measurements have determined that p-type material has a $\sim 20 \mathrm{x}$ higher free carrier absorption than equivalently doped n-type material. Thus it is imperative to minimize the doping density and thickness of any p-type layers in the structure for optimum optical performance. In addition, highly doping the LCL pushes the plasma reflection from a turn on of $>10 \mu \mathrm{m}$ for moderately doped InGaAs $\left(10^{18} / \mathrm{cm}^{3}\right)$ to a turn on in the $5 \mu \mathrm{m}$ to $8 \mu \mathrm{m}$ region (figure 3). Associated with the plasma turn on is an increase in the absorption, thus a heavily doped LCL degrades the optical performance by increasing the absorption in a region where there is appreciable photon flux.

A solution to the parasitic plasma absorption in the highly doped LCL was developed utilizing a novel, interdigitated interconnect scheme $[5,6]$. This design incorporated a more lowly LCL carrier concentration of $10^{18} / \mathrm{cm}^{3}$ rather than the $10^{19} / \mathrm{cm}^{3}$ carrier concentration used in the conventional MIM design. The lower doping level shifted the plasma absorption to wavelengths greater than $10 \mu \mathrm{m}$, thereby minimizing the deleterious absorption. Reduced LCL doping leads to an increased series resistance in this layer. The interdigitated contact grid (figure 4) increased metal coverage on the LCL and mitigated the problem of increased series resistance.

Monte Carlo photonic simulation was conducted and demonstrated that any disturbance from a planar first surface seriously degraded the spectral control performance of the MIM [7]. The interdigitated design, which incorporates v-groove trenches for electrical isolation (as does the conventional MIM design) as well as dovetail trenches for the LCL contact grid, suffered the greatest degradation in optical performance due to this effect. An additional interconnect design was developed (grid finger interconnect, figure 5) to allow more light into the device. The Monte Carlo simulations also demonstrated that allowing light to enter the MIM through the isolation trench reduced the optical performance of the MIM. Thus, current high performance MIMs all incorporate the buss bar interconnect in which a continuous reflective gold film covers the isolation trench. It has been demonstrated that reflecting the incident photons out of the interconnect region improves the optical performance.

The $\mathrm{p} / \mathrm{n}$ MIM achieved reasonable electrical and optical performance although the optical performance still required improvement. The primary optical loss mechanism was free carrier absorption in the heavily doped p-type emitter. The need to maintain a reasonable grid separation forced the emitters to be relatively thick and highly doped to minimize sheet resistance. A novel device design was introduced which minimized the amount of highly doped $\mathrm{p}$-type material, by utilizing an $\mathrm{n} / \mathrm{p}$ cell polarity and then incorporating a thin tunnel junction to allow the use of an n-type LCL (figure 6) $[8,9]$. This design has been called the TJ MIM or n/p/n MIM design. Besides the optical improvements, the design has several electrical advantages over the previous $\mathrm{p} / \mathrm{n}$ configuration. An n-type emitter has a much lower sheet resistance (25X), thus the series resistance of the MIM is 
reduced. In addition, the density of the emitter contact grid may be reduced thereby increasing the active area of the device. These electrical and optical improvements have been realized in the high efficiency device described below.

Up to this point, this discussion has been limited to lattice-matched $\operatorname{In}_{0.53} \mathrm{Ga}_{0.47} \mathrm{As}$ based MIM devices. Many applications require the use of lower bandgap photovoltaic devices, thus several organizations investigated the development of low bandgap MIM devices. Multiple buffer layer materials and designs have been investigated to accommodate the $>1 \%$ lattice mismatch between the desired $\mathrm{In}_{1-\mathrm{x}} \mathrm{Ga}_{\mathrm{x}} \mathrm{As}$ composition ( $\mathrm{x}=0.69$ for $0.6 \mathrm{eV}$ devices) and the semi-insulating InP substrate. To date, the preferred structure incorporates a compositionally stepped InPAs buffer layer [10 - 14]. Details of lattice mismatched MIM development are addressed in the paper in this issue by S. Murray.

Currently, the highest reported cell efficiency for a $0.6 \mathrm{eV} \mathrm{n} / \mathrm{p} / \mathrm{n}$ MIM (defined as output electrical power / incident convertible power) is $23.9 \%$ [15]. The MIM efficiency has been traditionally reported as the output electrical power / total absorbed power, which combines the energy conversion efficiency of the diode and spectral control efficiency of the MIM into a single performance indicator. The highest reported MIM efficiency is over $20 \%$ for a $0.6 \mathrm{eV} \mathrm{n} / \mathrm{p} / \mathrm{n}$ MIM [15]. This test was conducted on a $2 \mathrm{~cm} \times 2 \mathrm{~cm}, 30$ cell MIM illuminated by a gray body radiator at $1058^{\circ} \mathrm{C}$ with the cell held at $26.7^{\circ} \mathrm{C}$. The device structure is shown in figure 6 and produced $0.90 \mathrm{~W} / \mathrm{cm}^{2}$. It should be noted that illumination under a gray body radiator maximizes power density at the expense of efficiency. Improved efficiencies and reduced power densities may be possible if the device were tested under an appropriately designed selective emitter.

\section{Tandem $\operatorname{In}_{1-\mathrm{X}} \mathrm{Ga}_{\mathrm{X}} \mathrm{As}$ MIM}

Multiple bandgap, tandem converters can be used to enhance TPV system efficiency and power density. Improved over-excitation factors allow tandem devices to convert absorbed energy into useful power more efficiently than single-junction devices [16]. Stacking diodes in tandem produces a device with higher power density than that of a single-junction converter due to improved utilization of the incident spectrum. The common, semi-insulating substrate employed in $\operatorname{In}_{1-\mathrm{X}} \mathrm{Ga}_{\mathrm{X}} \mathrm{As}$ single-junction MIMs is directly applicable to $\operatorname{In}_{1-\mathrm{x}} \mathrm{Ga}_{\mathrm{X}} \mathrm{As}$ tandem MIMs.

The near perfect optical transparency of semi-insulating InP substrates to the spectrum of interest $\left(1200-1500 \mathrm{~K}\right.$ blackbody) and the applicability of $\operatorname{In}_{1-\mathrm{X}} \mathrm{Gax} A \mathrm{~s}$ to a wide range of bandgaps $(0.5-0.75 \mathrm{eV})$ supports an inverted tandem converter design [17]. In this design, the epilayers are deposited on one side of the InP substrate and the device is illuminated from the opposite side of the substrate. The subcell containing the diode of highest bandgap is first grown epitaxially on top of the substrate. Typically, $0.74 \mathrm{eV}$ $\mathrm{In}_{0.53} \mathrm{Ga}_{0.47} \mathrm{As}$ is the diode material of choice, as this composition has a lattice parameter identical to that of $\operatorname{InP}(5.8696 \AA)$. An interconnection region specific to the intended operation of the device is then grown, customarily using material of the same lattice parameter as the first subcell. The interconnection region consists of a tunnel junction in the case of series-connected (SC) tandem diodes or an isolation mechanism in the case of 
voltage-matched, or parallel-connected (PC), tandem diodes. A compositionally graded InAsP buffer is then employed to grade the lattice parameter of the crystal from that of the lattice-matched subcell to the lattice parameter corresponding to the target $\mathrm{In}_{1-\mathrm{X}} \mathrm{GaX} A \mathrm{As}$ composition. Finally, the low-bandgap diode is grown on top of this buffer. The components of such a tandem TPV structure are shown in figure 7.

The voltage of a TPV device varies linearly as radiator temperature changes, while the current varies exponentially. As a result, one advantage of a PC design over a SC design for tandem TPV applications is the relative insensitivity of the PC tandem device to variations in radiator temperature. While the $\mathrm{SC}$ case has commonly been considered for solar applications, the limited literature regarding tandem MIMs for TPV applications has focused on the PC case to date [18].

A two-terminal, PC, monolithic, tandem MIM is formed using a biaxially interconnected device architecture [19]. This design takes advantage of the two degrees of freedom available on the planar converter to yield a two-terminal, tandem device with two independent, orthogonal, MIMs (figure 8). The creation of a MIM within the individual subcells is accomplished using lateral conduction layers, isolation trenches, conduction metallization, and sidewall insulation, as in a single-junction MIM. Parallel interconnection of the independent MIMs requires that they be electrically isolated from one another. Isolation diodes [20] are one means to achieve this goal.

For a PC tandem device with two converters, voltage matching is achieved by adjusting the number of interconnected cells in each of the subcell MIMs so that the product of the number of cells times the maximum power point voltage per cell is equivalent for both strings. The two MIMs can then be connected in parallel on the edge of a die to form a monolithic, two-terminal device. The output power of the PC tandem MIM is then the sum of the power produced by each of individual MIMs.

The architecture and fabrication of a two-terminal SC tandem MIM does not differ substantially from that of a single-junction MIM. Additionally, the SC approach to tandem cell design and fabrication reduces material development needs (primarily due to the elimination of isolation diodes from the structure), and makes the extension of the tandem concept to triple and quadruple junction cells more straightforward. Because of these advantages, development of SC tandem MIMs for TPV can be useful for applications in which series connecting the tandem subcells has minimal impact on the device power production.

Spectral control considerations for a tandem MIM (PC or SC) are similar to those for a single-junction MIM. Some differences, however, do result from the exploitation of an inverted structure. The first surface of the device exposed to incident light is a featureless plane. As a result, light trapping due to the presence of grid fingers, interconnects, or etch trenches common to a standard single-junction MIM [7] is eliminated at the device surface. The planar surface also facilitates the direct deposition or attachment of a first surface filter to enhance transmission of above-bandgap photons and minimize transmission of below-bandgap photons. The back surface reflector in an inverted tandem 
MIM can be combined with the current-carrying conduction metal deposited on the epitaxial surface of the device. The ability to cover the epitaxial surface with metal without impacting device current production enables a structural design with a thinner emitter, lowering the reverse saturation current and raising the open-circuit voltage of the diode.

\section{InGaAsSb MIM}

An alternative to the more established InGaAs / InP based devices are InGaAsSb / GaSb based MIMs. InGaAsSb based TPV cells grown on GaSb substrates have been under development for some time and have shown electrical and optical properties comparable to the InGaAs / InP materials [21-23]. This quaternary material system is attractive due to the continuous range of bandgaps $(0.25-0.72 \mathrm{eV})$ that can be grown lattice matched to $\mathrm{GaSb}$, a commercially available substrate. Higher bandgap AlGaAsSb (0.72-1.65 eV) can also be grown lattice matched to GaSb. Consequently, complicated TPV structures incorporating windows, multiple junctions (as in the tandem cell), and quantum wells of various bandgaps can be grown without the strain relaxing buffer layers described earlier for the InGaAs / InP system.

However, in order to produce a MIM, a semi-insulating substrate is necessary, and GaSb is conductive due to a high number of intrinsic p-type carriers. Therefore some kind of isolation layer or layers must be grown between the GaSb substrate and the device layers, or the GaSb substrate must be removed after growth and replaced with an insulating one. This first kind of design, integrating epitaxial isolation into the device, is embodied with the use of a cell isolation diode (CID) [20]. A CID consists of a $\mathrm{p} / \mathrm{n}$ junction having reverse polarity with respect to the power producing diode as shown schematically in figure 9. The CID effectively acts to block current from leaking into the conductive GaSb substrate, allowing adjacent cells to be isolated and interconnected, allowing voltage building, the key feature of a MIM. As shown in figure 9, current MIMs consist of an InGaAsSb p/n photodiode, flanked by wider bandgap $\mathrm{GaSb}$ or $\mathrm{AlGaAsSb}$ window layers. The lateral conduction layer is highly doped $\mathrm{n}-\mathrm{GaSb}$ (the mobility of $\mathrm{n}-\mathrm{GaSb}$ is 5 6 times higher than that of $\mathrm{p}-\mathrm{GaSb}$ ) and the CID is a $\mathrm{n} / \mathrm{p}$ diode of either AlGaAsSb or $\mathrm{AlAsSb}$. The overall performance of this device is determined not just by the power production of the InGaAsSb diode, but just as importantly by the reverse breakdown and electrical leakage characteristics of the $\mathrm{Al}(\mathrm{Ga}) \mathrm{AsSb} \mathrm{CID}$. For this reason, a trade off will exist between the number and size of cells, which determines the operating voltage of the MIM device, and the required performance characteristics of the CID. Current state-ofthe-art AlGaAsSb and AlAsSb CIDs have been shown to have reverse breakdown voltages in the range of 5-7 volts [24]. The concept of a CID allowing voltage building for a MIM grown on a conductive substrate has recently been demonstrated, but large area, many junction ( $>10$ junction) devices like those shown routinely in the InGaAs / InP system have not [25]. The high number of intrinsic carriers in the GaSb substrate also affects the spectral control possibilities for epitaxially isolated devices. Because the free carrier absorption is so high, back surface spectral control with an IR mirror is not feasible. 
The second possibility for fabricating InGaAsSb / GaSb MIMs involves transfer of the epitaxially grown, power producing layers to an insulating substrate or substrate coated with insulating layers. An example of a MIM fabricated using such a wafer bonding / substrate removal technique is shown schematically in figure 10 . The power producing layers and LCL are grown inverted with respect to the epitaxially isolated devices described above. Next the insulating material and whatever bonding agent is necessary are applied to epitaxial side of the GaSb wafer. The device wafer is then bonded to a "handle wafer" that can also function as the insulation layer, or simply as a substrate for the device layers. Finally, the GaSb substrate and etch stop layer are etched away, leaving the device layers exposed and bonded to the insulating substrate. In addition to allowing the isolation of adjacent cells, the removal of the GaSb substrate enables back surface spectral control. Functioning MIMs have been produced using this kind of technique, employing $\mathrm{SiO}_{2}$ as the insulating layer, $\mathrm{Au}$ as the bonding agent and IR mirror, and GaAs as the "handle wafer" [26]. While epitaxial-transfer MIMs have shown promise by building voltage, they too have not yet been demonstrated with large area / many junction devices.

\section{Conclusions}

Monolithic Interconnected Module (MIM) development for thermophotovoltaic (TPV) energy conversion applications has been described. A variety of MIM devices have been developed using different materials, interconnecting schemes and designs. Current MIM performance provides encouragement that attractive TPV systems many soon be demonstrated. As with most TPV technologies, longevity of the MIM devices and the TPV systems are yet to be explored. Spin-off developments of the MIM design are being tested for a variety of non-TPV applications and in fact some are already flying in space [27].

\section{References}

[1] Wilt, D. M., et. al., "InGaAs PV Device Development for TPV Power Systems", Proc. $1^{\text {st }}$ NREL Thermophotovoltaic Generation of Electricity Conf., AIP 321, pp. 210-220, 1994.

[2] Wojtczuk, S., et. al., "p/n In( $\mathrm{Al}) \mathrm{GaAs}$ multi-junction laser power converters," Proc. $13^{\text {th }}$ Space Photovoltaic Research and Technology Conference, NASA Conference Publication 3278, p. 363, 1994.

[3] Wilt, D. M., et al., "Monolithically Interconnected InGaAs TPV Module Development," Proc. $25^{\text {th }}$ IEEE PVSC, May 1996, pp. 43-48.

[4] Wilt, D. M., et al., "Electrical and Optical Performance Characteristics of $0.74 \mathrm{eV}$ p/n MIM InGaAs Monolithic Interconnected Modules", Proc. $3^{\text {rd }}$ NREL Thermophotovoltaic Generation of Electricity Conf., AIP 401, pp. 237-247, 1997. 
[5] Ward, J. S. et. al., "A Novel Design for Monolithically Interconnected Modules (MIMs) for Thermophotovoltaic (TPV) Power Conversion," Proc. $3^{\text {rd }}$ NREL Thermophotovoltaic Generation of Electricity Conf., AIP 401, pp. 227-236, 1997.

[6] Ward, J. S., et. al., "Interdigitated Photovoltaic Power Conversion Device," U.S. Patent No. 5,897,715, 4/27/99.

[7] Ballinger, C. T., et. al., "Monte Carlo Analysis of a Monolithic Interconnected Module with a Back Surface Reflector," Proc. 4th NREL Thermophotovoltaic Generation of Electricity Conf., AIP 460, pp. 161- 174, 1998.

[8] Murray; C. S., et. al., "Monolithic interconnected module with a tunnel junction for enhanced electrical and optical performance", U.S. Patent No. 6,162,987, $12 / 19 / 00$

[9] Wilt, D. M., et. al., " $\mathrm{n} / \mathrm{p} / \mathrm{n}$ Tunnel Junction InGaAs Monolithic Interconnected Module (MIM)", Proc. 4th NREL Thermophotovoltaic Generation of Electricity Conf., AIP 460, pp. 152- 160, 1998.

[10] Hoffman, R., et. al., "Using a Critical Composition Grading Technique to Deposit InGaAs Epitaxial Layers on InP Substrates," Patent pending.

[11] Fatemi, N. S., et. al., "High-Performance Lattice-Mismatched InGaAs/InP MIMs," Proc. 4th NREL Thermophotovoltaic Generation of Electricity Conf., AIP 460, pp. 121- 131, 1998.

[12] Wilt, D. M., et al., "0.55-eV n/p/n MIM TPV Cell Development," Proc. $28^{\text {th }}$ IEEE PVSC, September 2000, pp. 1024-1027.

[13] Wanlass, M. W., et. al., "High-Performance, 0.6-eV $\mathrm{Ga}_{0.32} \mathrm{In}_{0.68} \mathrm{As}_{\mathrm{S}} / \mathrm{InAs}_{0.32} \mathrm{P}_{0.68}$ Thermophotovoltaic Converters and Monolithically Interconnected Modules," Proc. 4th NREL Thermophotovoltaic Generation of Electricity Conf., AIP 460, pp. 132- 141, 1998.

[14] Wanlass, M. W., "Low-Bandgap, Double-Heterostructure InAsP/GaInAs Photovoltaic Converter," U. S. Patent No. 6,300,557, 10/9/01.

[15] Sergiej, R. R., et al, "20\% Efficient InGaAs/InPAs Thermophotovoltaic Cells," Proc. $5^{\text {th }}$ TPV Conf, AIP, September 2002.

[16] Fahrenbruch, A. L., and Bube, R. H., "Concentrators, Concentrator Systems, and Photoelectrochemical Cells," in Fundamentals of Solar Cells, Academic Press, Inc., San Diego, 1983, pp. 520-526. 
[17] Wanlass, M. W., et. al., "Low-Bandgap, Monolithic, Multi-Bandgap, Optoelectronic Devices," Patent pending.

[18] Wehrer, R. J., et al., “0.74/0.55-eV Ga $\operatorname{In}_{1-\mathrm{X}} \mathrm{As} / \mathrm{In} \mathrm{As}_{\mathrm{Y}} \mathrm{P}_{1-\mathrm{Y}}$ Monolithic, Tandem, MIM TPV Converters: Design, Growth, Processing and Performance," Proc. $29^{\text {th }}$ IEEE PVSC, May 2002, to be published.

[19] Wanlass, M. W., et. al., "Voltage-Matched, Monolithic, Multi-Bandgap Devices," Patent pending.

[20] Wanlass, M. W., "Electrical Isolation of Component Cells in Monolithically Interconnected Modules," U. S. Patent No. 6,239,354, 5/29/01.

[21] Wang, C.A., "Extending the Cutoff Wavelength of Lattice Matched GaInAsSb / GaSb Thermophotovoltaic Devices," Proc. 4th NREL Thermophotovoltaic Generation of Electricity Conf., AIP 460, pp. 256- 265, 1998.

[22] Wang, C.A., et al., "Recent progress in GaInAsSb thermophotovoltaics grown by Organometallic vapor-phase epitaxy," J. Crystal Growth 195, 346-355 (1998).

[23] Martinelli, R.U., Garbuzov, D.Z., Lee, H., Morris, N., Odubanjo, T., Taylor, G.C., and Connolly, J.C., "Minority-Carrier Transport in InGaAsSbThermophotovoltaic Diodes," Proc. $3^{\text {rd }}$ NREL Thermophotovoltaic Generation of Electricity Conf., AIP 401, 389-395, 1997.

[24] Cederberg, J.G., et al, "The Preparation of $\mathrm{InGa(As)Sb}$ and $\mathrm{Al}(\mathrm{Ga}) \mathrm{AsSb}$ Films and Diodes on GaSb for Thermophotovoltaic Applications Using Metal Organic Chemical Vapor Deposition," accepted for publication in J. Crystal Growth (2002).

[25] Palmisiano, M.N., et al, "Development of InGaAsSb Based Monolithic Interconnected Modules," Proc. $5^{\text {th }}$ TPV Conf, AIP, September 2002.

[26] Wang, C.A., et al., "Wafer-Bonded Internal Reflectors for Enhanced TPV Performance," Proc. $5^{\text {th }}$ TPV Conf, AIP, September 2002.

[27] Raffaelle, R. P., et al, "Microsat Power Supplies," Proc. 40 ${ }^{\text {th }}$ AIAA Aerospace Sciences Meeting, January 2002. 


\section{Captions for figures}

Figure 1 - a) A fifteen (15) cell, $1 \mathrm{~cm}^{2}$ MIM. b) A cross-sectional view of the original $\mathrm{p} / \mathrm{n}$ MIM design.

Figure 2 - Spectral absorption measurements for $3.0 \mu \mathrm{m} \mathrm{In}{ }_{0.53} \mathrm{Ga}_{0.47}$ As epilayers of various doping types and densities [4]. As the carrier concentration increases, the material absorbs more non-convertible photons. Known as increasing free carrier absorption (FCA), this phenomenon is illustrated here for p-type material. The addition of a high concentration of carriers shifts the bandgap of the material to shorter wavelengths. Known as the Burstein-Moss (B-M) shift, this effect is illustrated here in ntype material.

Figure 3 - Reflectance of $0.74 \mathrm{eV}$ MIM (without ARC). The plasma reflectance at a wavelength of $\sim 8 \mu \mathrm{m}$ occurs due to absorption in the highly doped LCL.

Figure 4 - Optical micrograph image showing the interdigitated interconnect design. The contact grid employed in this design decreases device series resistance.

Figure 5 - a: Optical micrograph image showing the grid finger interconnect design. This contact pattern reduces metal coverage on the device surface and increases device active area. b: Enlargement of the interconnect area.

Figure 6 - A cross-sectional view of the tunnel junction (n/p/n) MIM design.

Figure 7 - The basic components of an inverted, tandem MIM.

Figure 8 - Biaxial, tandem MIM device designed to voltage-match the individual, parallel-connected converter strings (MIMs). The low-bandgap and high-bandgap converter strings (10 and 5 diodes, respectively) are orthogonally oriented with respect to one another.

Figure 9 - Wiring diagram (left) and structure (right) of CID based $\mathrm{InGaAsSb} / \mathrm{GaSb}$ MIM

Figure 10 - Schematic of epitaxial transfer technique for MIM fabrication on conductive GaSb substrate showing (in order) wafer bonding, substrate removal, and MIM fabrication. 
Figure 1

a)

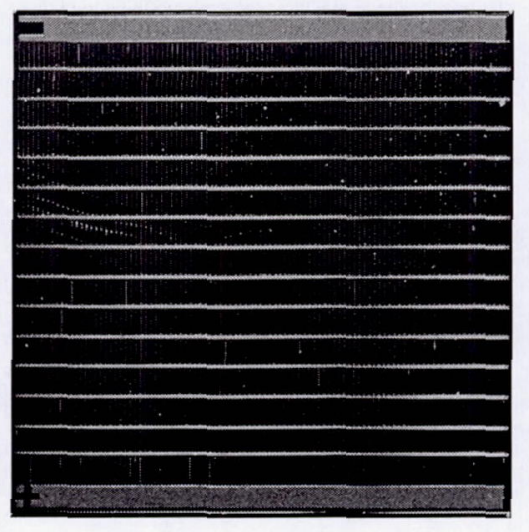

b)

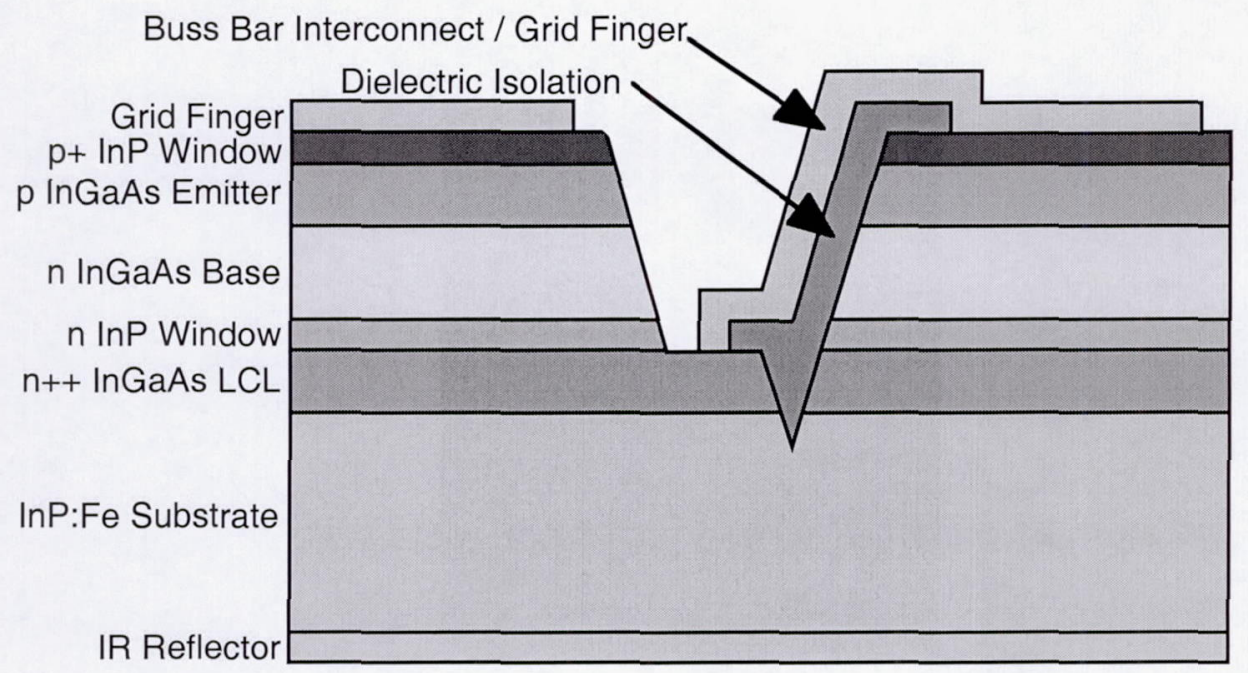


Figure 2

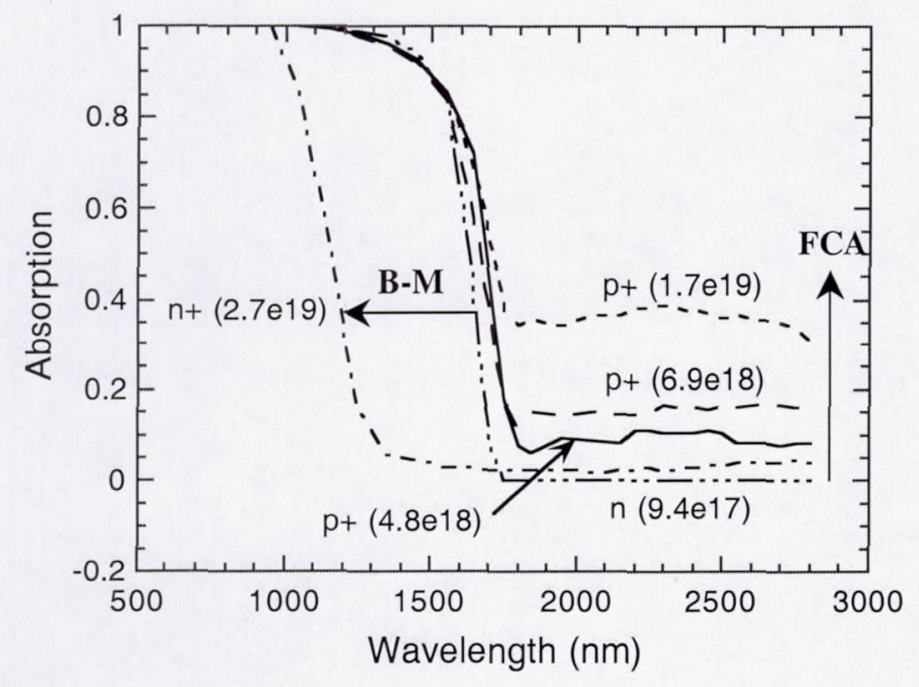


Figure 3

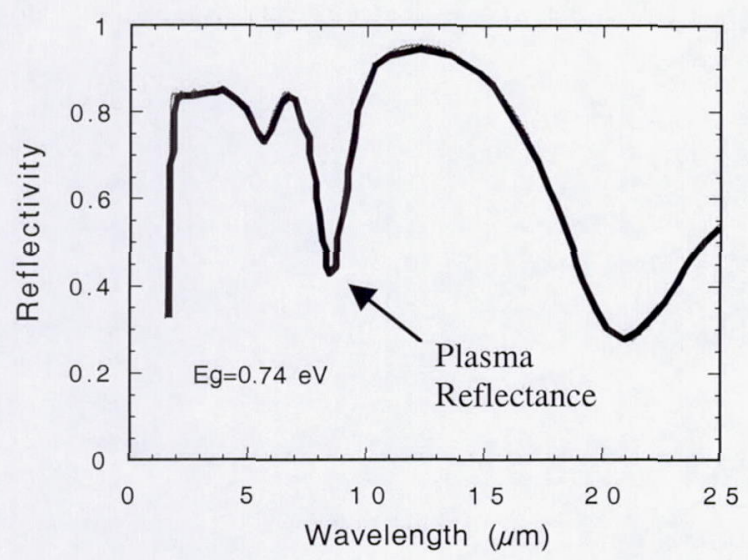


Figure 4

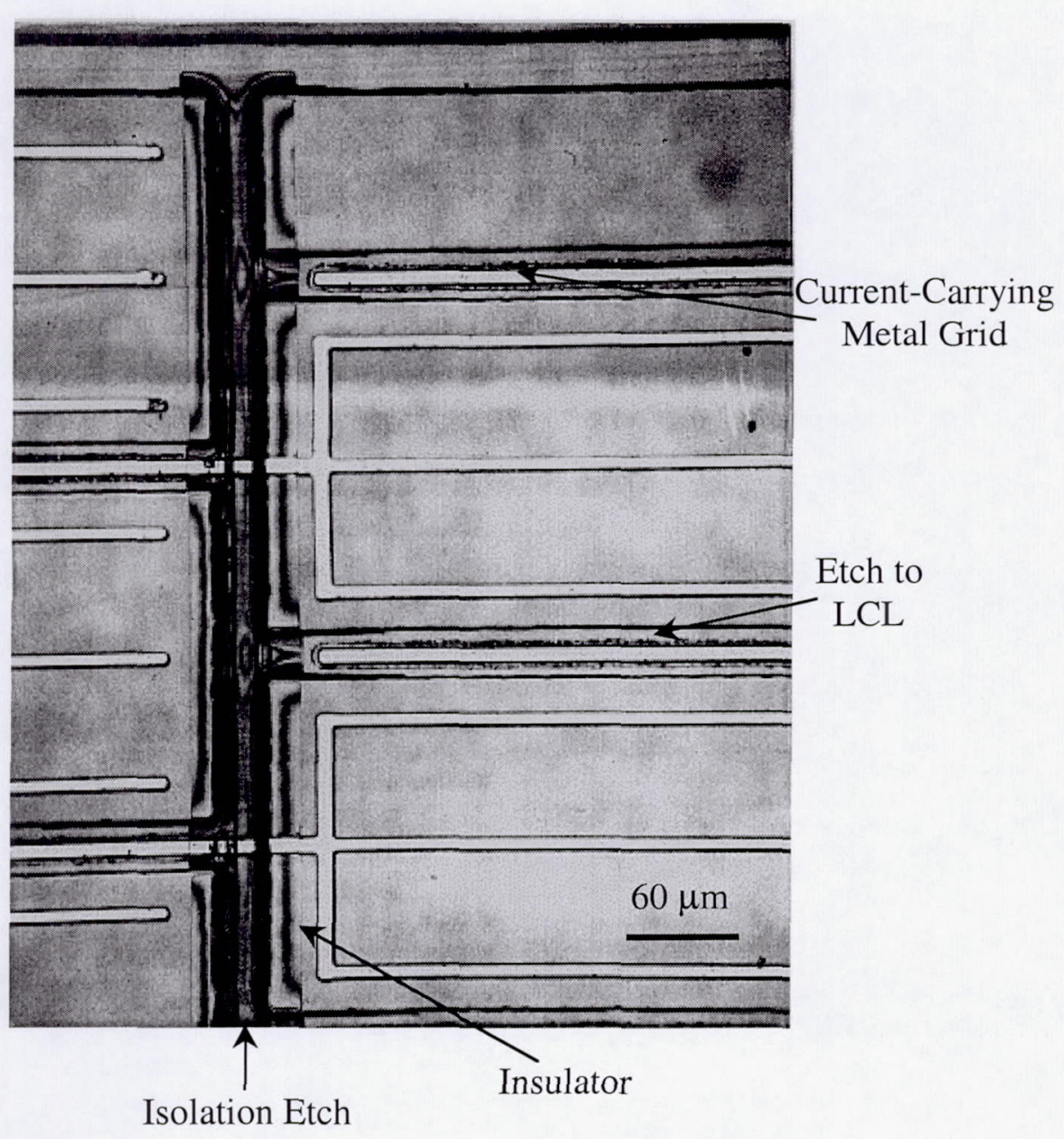


Figure $5 \mathrm{a}$ and $\mathrm{b}$
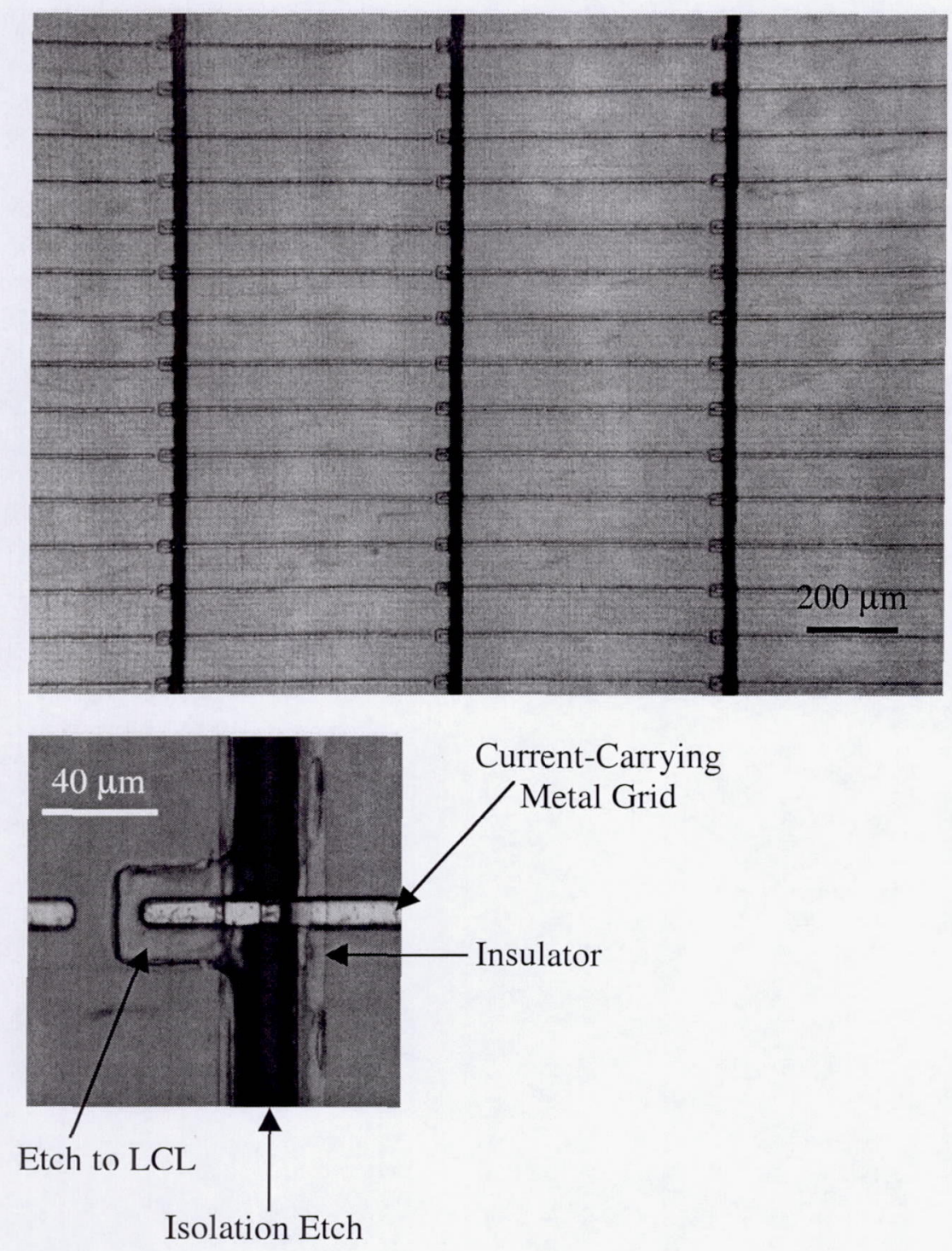
Figure 6

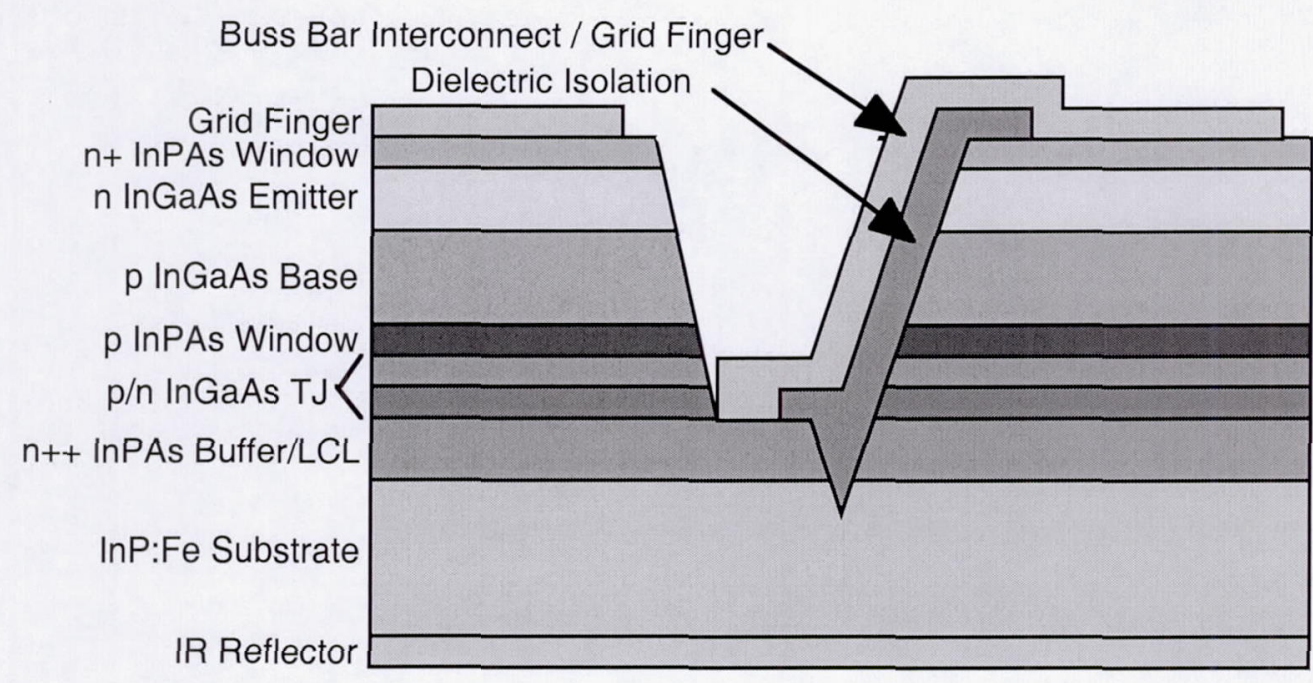


Figure 7

Illumination through transparent InP substrate

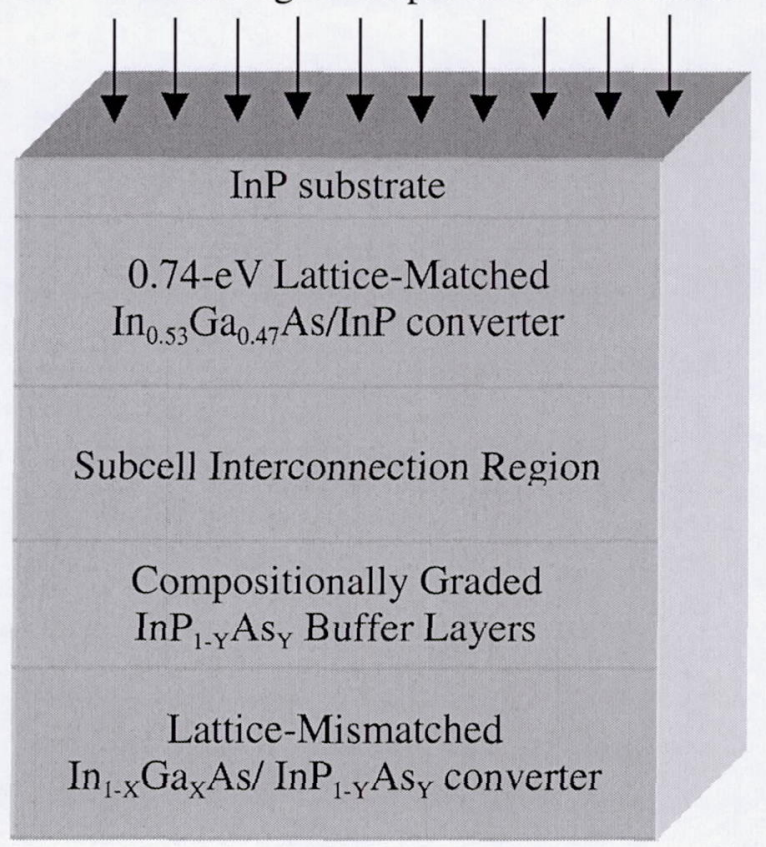


Figure 8

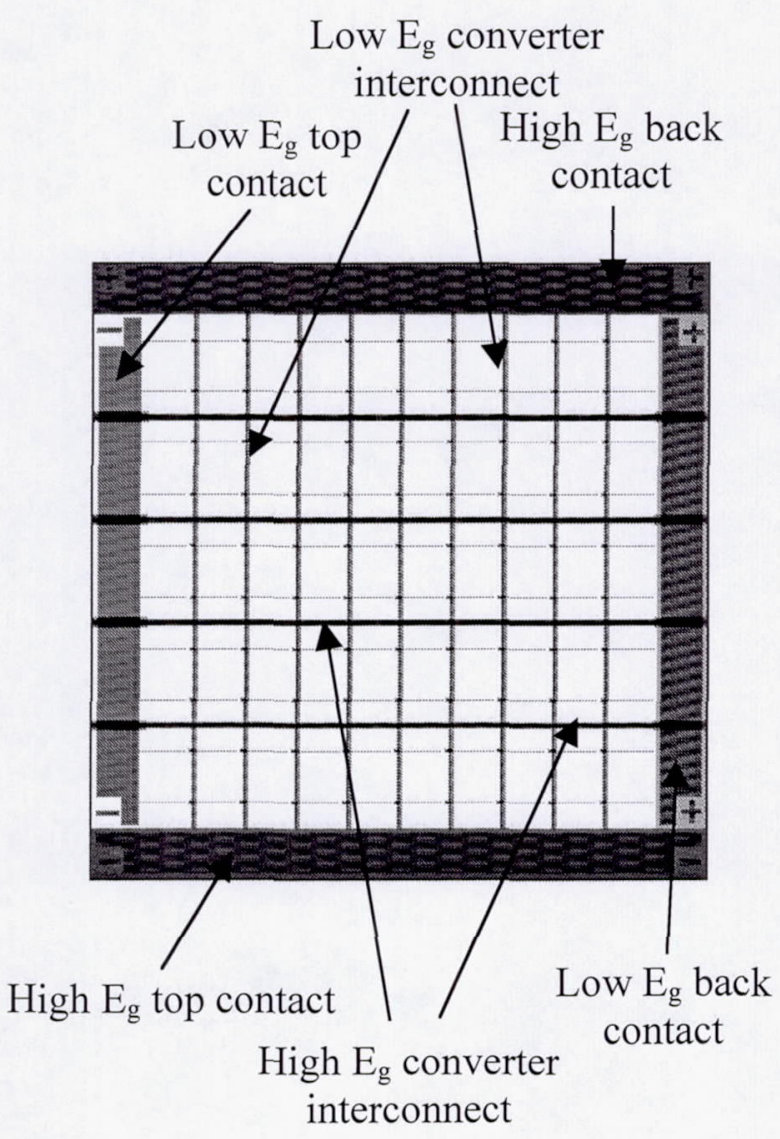


Figure 9

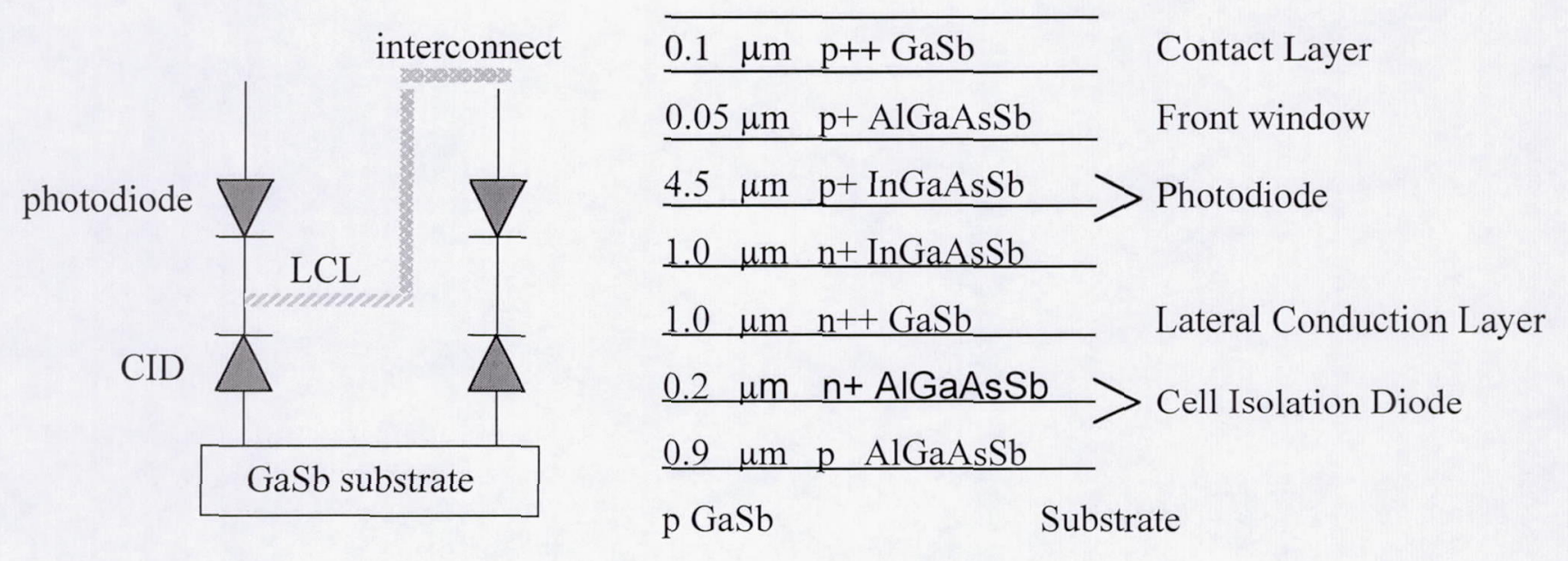


Figure 10

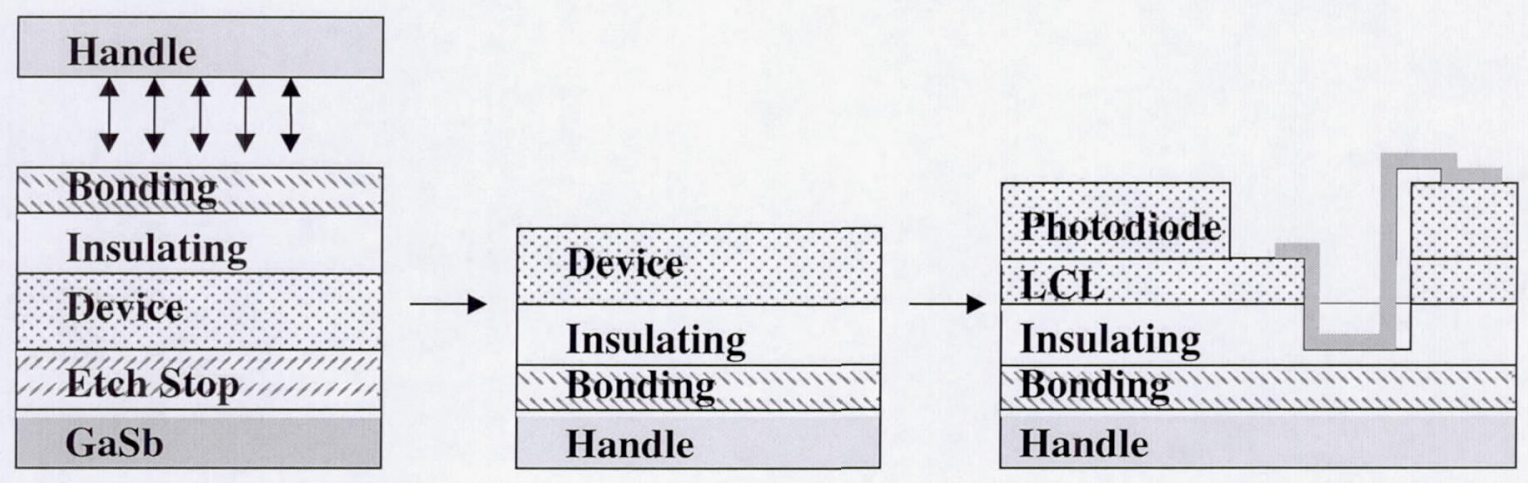

